\title{
WILEY-VCH
}

DOI: $10.1002 /(($ please add manuscript number $))$

Article type: Communication

\section{Partially Reduced Holey Graphene Oxide as High-Performance Anode for Sodium-Ion Batteries}

Jin Zhao, Yi-Zhou Zhang, Fan Zhang, Hanfeng Liang, Fangwang Ming, Husam N. Alshareef* and Zhiqiang Gao*

Dr. J. Zhao, Prof. Z. Gao

Department of Chemistry, National University of Singapore, 117543, Singapore

E-mail: chmgaoz@hotmail.com

Dr. Y. Z. Zhang, F. Zhang, Dr. H. Liang, F. Ming, Prof. H. N. Alshareef

Materials Science and Engineering, Physical Science and Engineering Division, King Abdullah University of Science and Technology (KAUST), Thuwal, 23955-6900, Saudi Arabia

E-mail: husam.alshareef@kaust.edu.sa

Keywords: Holey graphene oxide; Anode material; Energy storage; Sodium-ion batteries; Oxygenous groups

Abstract: The current $\mathrm{Na}^{+}$storage performance of carbon-based materials is still hindered by the sluggish $\mathrm{Na}^{+}$ions transfer kinetics and low capacity. Graphene and its derivatives have been widely investigated as electrode materials in energy storage and conversion systems. However, as anode materials for sodium-ion batteries (SIBs), the severe $\pi-\pi$ restacking of graphene sheets usually results in compact structure with a small interlayer distance and a long ion transfer distance, thus leading to low capacity and poor rate capability. Herein, partially reduced holey graphene oxide is prepared by simple $\mathrm{H}_{2} \mathrm{O}_{2}$ treatment and subsequent low temperaturereduction of graphene oxide, leading to large interlayer distance $(0.434 \mathrm{~nm})$, fast ion transport and more $\mathrm{Na}^{+}$storage space. The partially remaining oxygenous groups can also contribute to the capacity by redox reaction. As anode material for SIBs, the optimized electrode delivered high reversible capacity, high rate capability (365 and $131 \mathrm{mAh} \mathrm{g}^{-1}$ at 0.1 and $10 \mathrm{~A} \mathrm{~g}^{-1}$, respectively) and good cycling performance ( $163 \mathrm{mAh} \mathrm{g}^{-1}$ after 3000 cycles at a current density of $2 \mathrm{~A} \mathrm{~g}^{-1}$ ), among the best for carbon-based sodium ion battery anodes. 


\section{WILEY-VCH}

Developing advanced rechargeable batteries as energy storage devices for renewable energy sources is of critical importance to addressing the energy and environmental crises facing humanity today. Sodium-ion batteries (SIBs) are a promising energy storage system beyond lithium-ion batteries, which have attracted significant attention due to the earth abundance, wide availability of sodium resources and the similar physicochemical properties of sodium and lithium. ${ }^{[1]}$ However, since the radius of sodium ion is $55 \%$ larger than that of lithium ion, the direct use of electrode materials developed for lithium-ion batteries in SIBs usually results in low capacity, low rate capability, and poor stability. Thus, the development of appropriate host materials with sufficient interlayer spacing for sodium ion accommodation and fast insertion/extraction is crucial. ${ }^{[2]}$ Fortunately, many materials such as layered transition metal oxides and polyanionic compounds have been demonstrated as promising cathode materials for SIBs ${ }^{[3]}$ The search for anode materials for SIBs, however, has long lagged behind and greatly hindered the commercialization of SIBs ${ }^{[4]}$ In recent years, much progress has been achieved in the field for preparation of 2D materials and their composites, which provides a wide range of choices for developing advanced anodes for SIBs. ${ }^{[1,5]}$

Among reported anode materials for SIBs, carbon-based materials have been widely investigated and are regarded as the most promising anode materials for SIBs. This is due to the features of high conductivity, abundance, easy modification of their structures, low cost, as well as the relatively small volume expansion during sodiation. ${ }^{[4 a, 6]}$ Among various carbonbased materials, graphene with the features of large theoretical specific surface area and high conductivity has been widely studied as possible electrode material in energy storage and conversion systems, such as supercapacitors, lithium-ion batteries, lithium-sulfur batteries, fuel cells, and so on. ${ }^{[7]}$ Unfortunately, as for SIBs, the severe $\pi-\pi$ restacking of graphene sheets usually leads to a compact uniaxially oriented stacked structure with a small interlayer distance, thus severely impacting the capacity and rate capability of SIBs ${ }^{[8]}$ Although a few approaches 


\section{WILEY-VCH}

have been proposed to address this issue, such as nitrogen doping, introducing spacer and creating crumples, the energy storage capability of graphene-based materials for sodium ions is still far from satisfactory, especially at high operating rates. ${ }^{[8 c, 9]}$

The morphology of carbon-based materials plays an important role in determining its energy storage performance by altering the ion transfer behavior. ${ }^{[10]}$ Tuning morphology has been demonstrated to be effective in improving the energy storage capability of carbon-based materials. ${ }^{[11]}$ It has recently been demonstrated that creating holey graphene with a hierarchical porous structure, i.e., with large-sized pores (macropores) in the 3D network and micro- to meso-pores in the graphene sheets is an effective approach to improve the high rate performance of graphene. ${ }^{[12]}$ On the other hand, heteroatom (e.g. N, O, S, B, P and etc.) functionalization is an effective strategy to modulate the physicochemical properties such as conductivity and wettability of carbon-based materials, as well as to increase capacity via redox reactions. ${ }^{[13]}$

In this report, partially reduced holey graphene oxide (HRGO) was prepared by a simple energy efficient thermo-reduction process of the holey graphene oxide (GO) under relatively low temperature. The advantages of the HRGO for sodium ion storage include: (i) The holes on the partially reduced holey graphene oxide sheets increase the insertion/extraction rate by shortening the ion transfer distance and providing more sodium ion insertion/extraction sites. (ii) The residual oxygen-containing functional groups can serve as the spacer to prevent severe $\pi-\pi$ restacking of graphene sheets and to ensure a large interlayer distance, which provides sufficient storage space for sodium ions and facilitates the fast transfer of sodium ions. (iii) The residual oxygen-containing functional groups also contribute to the energy storage capability via redox reactions. With these favorable features, the HRGO prepared at $300{ }^{\circ} \mathrm{C}$ in this work exhibited a high storage capability with large capacity (365 mAh g $\mathrm{m}^{-1}$ at $\left.0.1 \mathrm{~A} \mathrm{~g}^{-1}\right)$, high rate capability (131 mAh g $\mathrm{m}^{-1}$ at $\left.10 \mathrm{~A} \mathrm{~g}^{-1}\right)$ and good cycling stability.

Scheme 1 depicts the preparation process of HRGO and graphene under $300^{\circ} \mathrm{C}$, named 


\section{WILEY-VCH}

as HRGO300 and RGO300, respectively, as well as the ion transfer channels in them. Briefly, GO is etched with diluted hydrogen peroxide to form holey GO. Then, the holey GO is reduced under $300{ }^{\circ} \mathrm{C}$ to produce $\mathrm{HRGO} 300$ interlayered with partially remaining oxygen-containing groups. The control material, RGO300, is prepared by treating GO with the above-mentioned process without hydrogen peroxide etching (Scheme 1a). The HRGO300 with enlarged interlayer distance can provide more ion insertion/extraction sites and shorter ion transfer distance than those of the RGO300, which are beneficial for high rate energy storage through sodium ions (Scheme 1b, c).

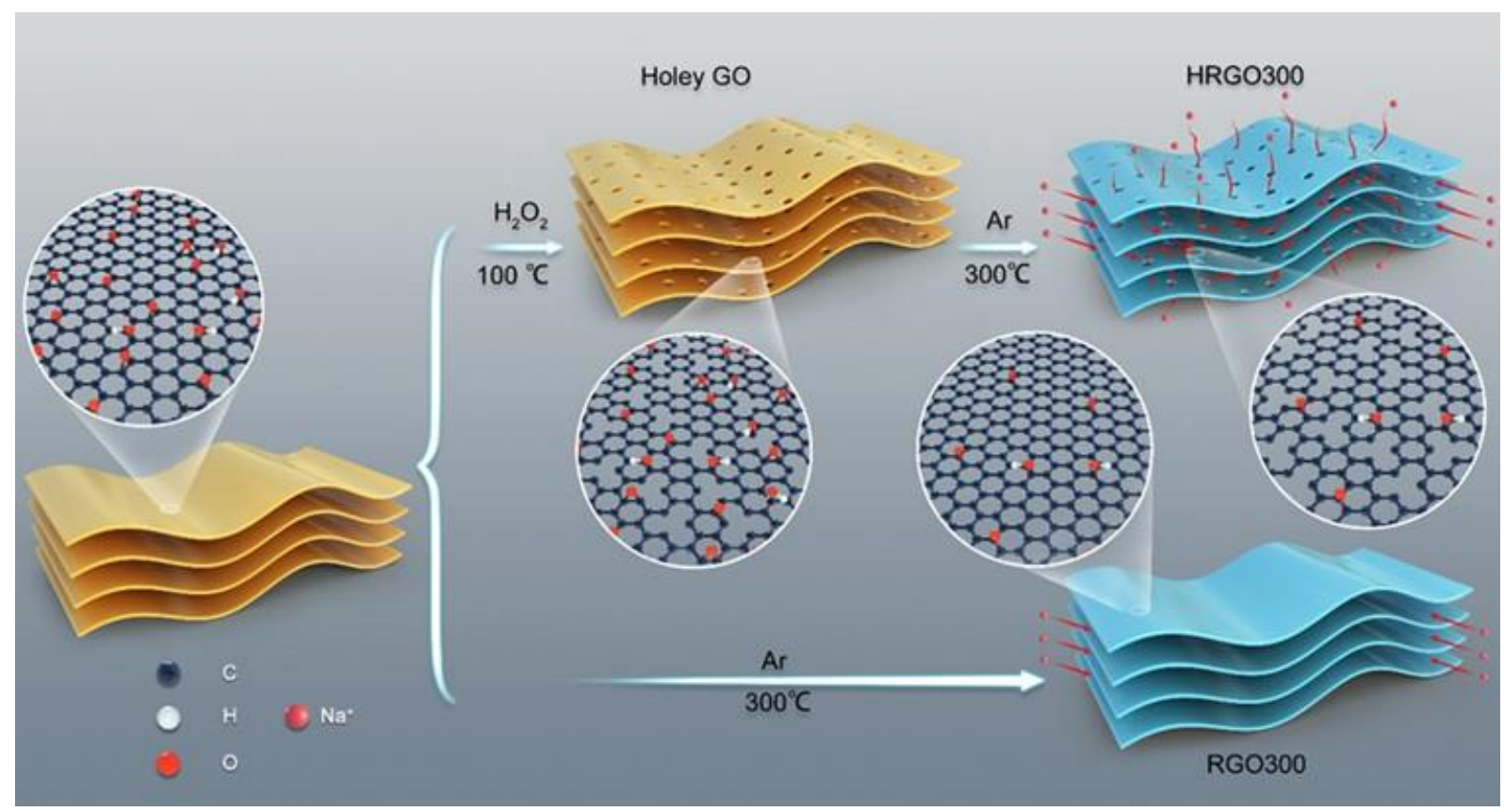

Scheme 1. Schematic illustration of the preparation of HRGO300 and RGO300. a) the preparation processes; b and c) ion transfer channels in HRGO300 (b) and RGO300 (c).

Figure 1 shows the morphology and microstructure of HRGO300 and RGO300. As shown in the SEM images, HRGO300 and RGO300 both possess a flake-like structure of several hundred microns in size, but HRGO300 is more crumpled than RGO300 (Figure 1a and d). Furthermore, TEM observations clearly indicate the abundant nanometer-sized pores on HRGO300 sheets (Figure 1b), in sharp difference from the relatively intact feature of RGO300 (Figure 1e), suggesting that the partially reduced graphene oxide sheets have been effectively 


\section{WILEY-VCH}

etched by hydrogen peroxide (Figure $1 \mathrm{~b}$ and e). No obvious morphology changes can be observed in the TEM images of RGO300 captured at different magnifications and exposure time, suggesting the pores on HRGO300 are not generated by electron beam irradiation (Figure S1, Supporting Information). During the charge/discharge process, the intact partially reduced graphene oxide sheets can only provide their edges as ion insertion/extraction sites. On the other hand, as for the HRGO sheets, the pores can provide significantly more ion insertion/extraction sites besides the edges and can shorten the ion transfer distance, which is favorable for high rate performance (Scheme 1). The high resolution TEM (HRTEM) images reveal that the HRGO300 is thicker than RGO300 (Figure 1c and f), which may be explained as the partially reduced graphene oxide sheets have already restacked to some extent when heated at $100{ }^{\circ} \mathrm{C}$ for $4 \mathrm{~h}$ during hydrogen peroxide etching. The average interlayer distances of HRGO300 and RGO300 are $\sim 0.427$ and $\sim 0.416 \mathrm{~nm}$, respectively (Figure $1 \mathrm{c}$ and f). The HRGO products prepared at 200 and $400{ }^{\circ} \mathrm{C}$ have the same crumpled flake-like morphology as HRGO300 with interlayer distances of 0.438 and $0.422 \mathrm{~nm}$, respectively (Figure S2, Supporting Information). All these values are larger than the $0.34 \mathrm{~nm}$ of pristine graphite and the theoretical electrochemically active interlayer distance of $>3.7 \mathrm{~nm}$ for sodium ion insertion/extraction in carbon-based materials, thus can increase capacity and rate capability. ${ }^{[14]}$ 


\section{WILEY-VCH}

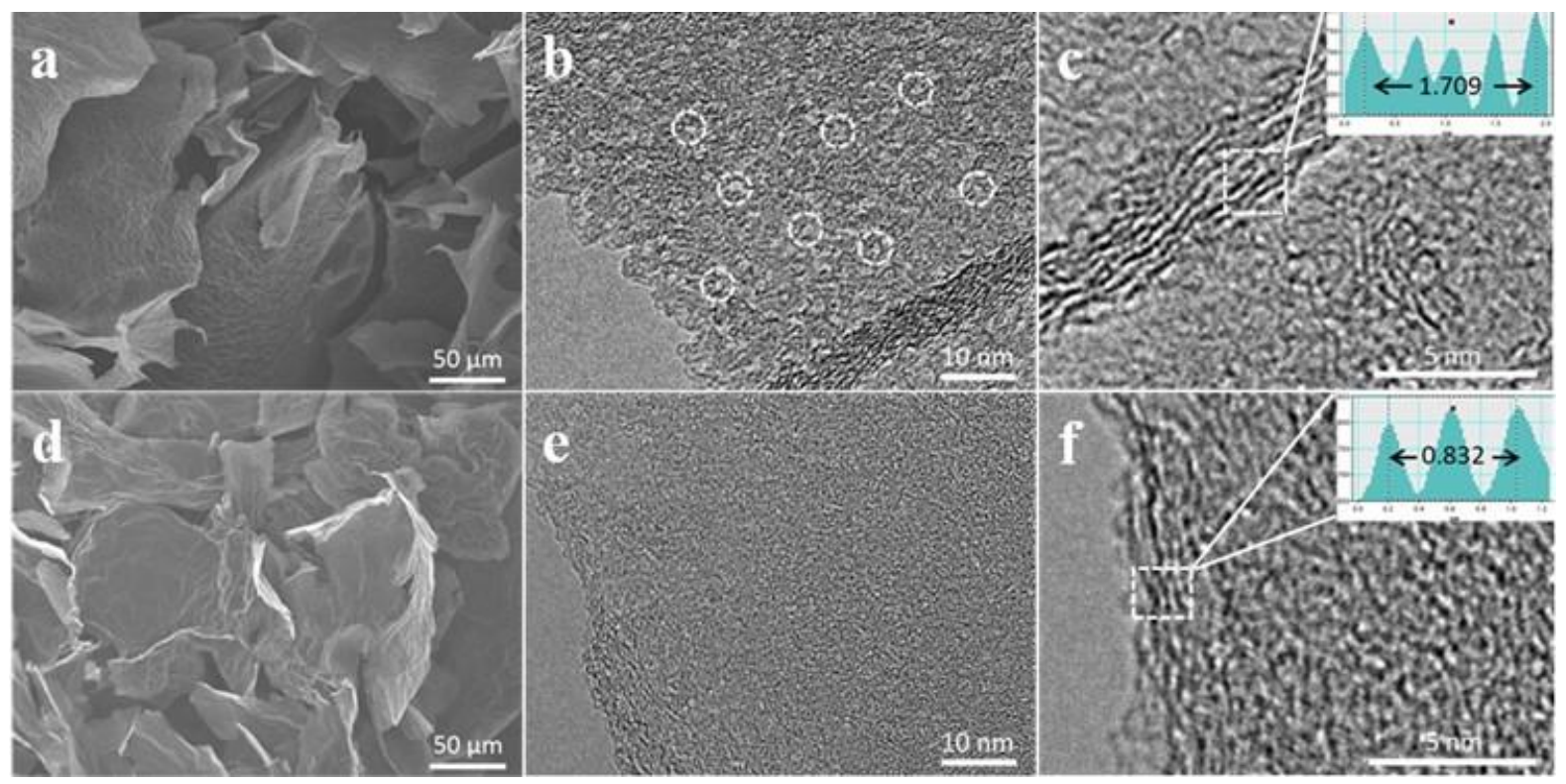

Figure 1. Morphological characterization of HRGO300 (a-c) and RGO300 (d-f). a and d) SEM images. $b$ and e) TEM images, the white circles in (b) indicate some of the pores. $c$ and f) HRTEM images. Insets: the corresponding intensity profiles for the line scan across the lattice fringes.

As presented in Figure 2, the structure of the products was further investigated by XRD, XPS and $\mathrm{N}_{2}$ sorption isotherms. All XRD patterns exhibit two broad peaks of (002) and (100) at $\sim 23^{\circ}$ and $43^{\circ}$, respectively, suggesting the amorphous nature of HRGO. ${ }^{[15]}$ The $(002)$ peak center at $21.39^{\circ}, 20.12^{\circ}, 20.46^{\circ}$, and $20.79^{\circ}$ for RGO300, HRGO200, HRGO300, and HRGO400, respectively, corresponding to the interlayer distance of $0.415,0.441,0.434$, and $0.427 \mathrm{~nm}$, respectively. The XRD results are consistent with the HRTEM observations (Figure 2a, Figure 1c, f and Figure S2 in Supporting Information). With increasing heat treatment temperature, the oxygen content in HRGO decreased gradually, which is responsible for the observed interlayer distance order of HRGO200 $>$ HRGO300 $>$ HRGO400 (Figure 2b). ${ }^{[14 b]}$ RGO300 shows smaller interlayer distance than HRGO300 and HRGO400 though its oxygen content is higher than those for HRGO300 and HRGO400. This can be ascribed to the much more crumpled structure and significant amount of defects in HRGO, which will weaken the strong $\pi-\pi$ interaction between the graphene sheets and thus increase the interlayer distance. ${ }^{[16]}$ A higher D/G intensity ratio in Raman spectra was observed in HRGO products than RGO300, 


\section{WILEY-VCH}

indicating of more defects in HRGO products. These defects are likely formed during the pore formation process (Figure S3, Supporting Information). The oxygen contents in the four heat treated samples are much smaller than 30.46 at.\% in the original graphene oxide, suggesting the effective removal of oxygenous groups under low temperature treatment (Figure $2 b$ and Figure S4 in Supporting Information). The oxygenous groups in the products exist in the form of ${ }^{-} \mathrm{C}^{-} \mathrm{O}^{-},{ }^{-} \mathrm{C}=\mathrm{O}$, and ${ }^{-} \mathrm{COO}^{-}$(Figure $\mathrm{S} 5$, Supporting Information). The partially remaining oxygenous groups can not only increase the intercalation-based energy storage through sodium ions by expanding the interlayer distance of HRGO, but can also contribute to energy storage by redox reactions. ${ }^{[11 a, 17]}$ All the adsorption/desorption isotherms of the products are type IV isotherms. However, the isotherm of HRGO300 shows an obvious steep uptake at $p / p_{0}<0.01$ and a much more obvious type $\mathrm{H} 3$ hysteresis loop at high pressure region $\left(p / p_{0}>0.4\right)$ as compared with those for HRGO products. This indicates that RGO300 has a loose structure with abundant micropores and slit-like meso-macropores (Figure 2c), which can be vividly demonstrated by the sharp contrast in volume of HRGO300 and RGO300 (Figure S6a in Supporting Information). ${ }^{[18]}$ The slit-like large meso- and macropores in RGO300 originate from the intra-aggregates of partially reduced graphene oxide sheets. After dispersing and drying, these aggregate pores can be removed, thus will not affect the performance of prepared electrode (Figure S6b in Supporting Information). The specific surface areas are 156, 71, 84 and $87 \mathrm{~m}^{2} \mathrm{~g}^{-1}$ for RGO300, HRGO200, HRGO300 and HRGO400, respectively. The disappearance of steep uptake at $p / p_{0}<0.01$ and the lower specific surface area of HRGO are likely attributed to the selectively etched amorphous carbon, which has more micropores and higher nitrogen adsorption capacity than the graphitic carbon. ${ }^{[11 \mathrm{~d}]}$ The slightly increased specific surface area of HRGO with higher heat treatment temperature is likely due to the fact that more oxygen-containing groups are removed at higher temperatures. ${ }^{[19]}$ As shown in the pore size distribution curves of HRGO products, the micropores in the range of 0.5 to $0.8 \mathrm{~nm}$ shown in RGO300 disappeared and more abundant mesopores with size of $\sim 2-10 \mathrm{~nm}$ appeared. 


\section{WILEY-VCH}

Again, this is consistent with the TEM observations (Figure $2 \mathrm{~d}$ and Figure $1 \mathrm{~b}$ and e), thus implying that the intrinsic micropores below $0.8 \mathrm{~nm}$ are enlarged after $\mathrm{H}_{2} \mathrm{O}_{2}$ etching.
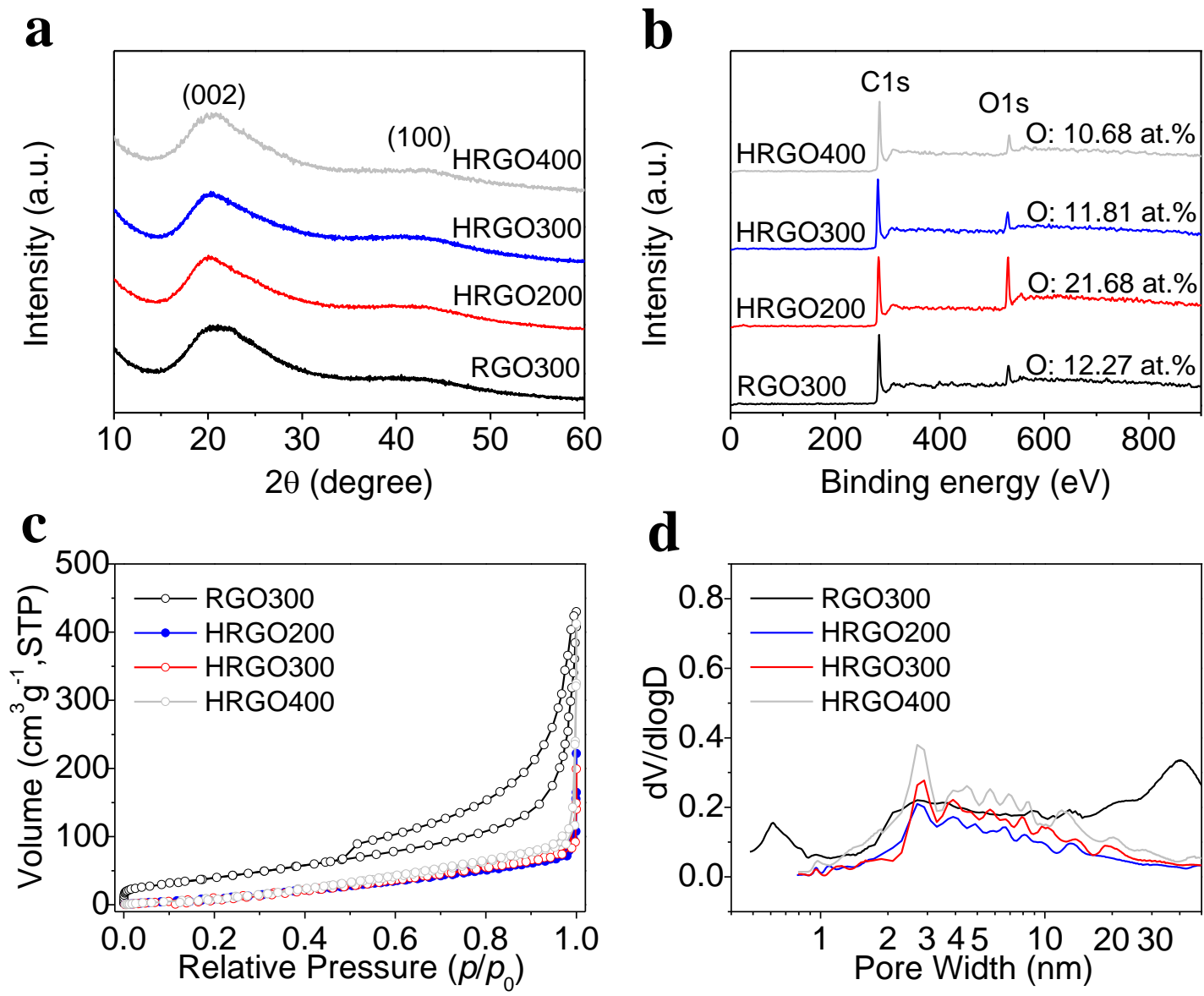

Figure 2. Microstructure analysis of the electrodes. a) XRD patterns, b) XPS spectra, c) Nitrogen adsorption/desorption isotherms, d) pore size distribution curves.

To evaluate the energy storage capability of HRGO products, 2025 coin-type cells were fabricated. Figure 3 shows the electrochemical performance of HRGO300 and RGO300 electrodes. In the cyclic voltammetric (CV) curves of HRGO300 measured at $0.1 \mathrm{mV} \mathrm{s}^{-1}$, the current peaks at around 2.25 and $1.0 \mathrm{~V}$ in the first cathodic process, but disappear in subsequent cycles, which can be ascribed to the decomposition of electrolyte, formation of solid electrolyte interphase (SEI) layer and irreversible side reactions between sodium ions and surface functional groups. ${ }^{[20]}$ The sharp cathodic peaks below $0.5 \mathrm{~V}$ are attributed to the sodium ion insertion into the layers/pores of the HRGO300 electrode. During the anodic process, no obvious peaks can be observed, suggesting that the sodium ion extraction process happens over 


\section{WILEY-VCH}

a broad potential range. ${ }^{[9 a, 21]}$ After the second cycle, the CV curves almost overlap with each other, suggesting that the sodiation-desodiation process is highly reversible (Figure 3a). The approximately rectangular-like CV curves after the first cycle imply considerable capacitive nature of the sodium storage behavior. ${ }^{[22]}$ The charge/discharge profiles of HRGO300 at a current density of $0.1 \mathrm{~A} \mathrm{~g} \mathrm{~g}^{-1}$ are different from those of typical hard carbon materials, which show a low-potential plateau close to $0 \mathrm{~V}$ (Figure 3b). The absence of the low-potential plateau helps to alleviate the safety problem in our devices. ${ }^{[3 c, 9 c]}$ The large irreversible capacity in the first cycle arises from the formation of the SEI layer and some irreversible side reactions. ${ }^{[23]}$ After several cycles of activation at the current density of $0.1 \mathrm{~A} \mathrm{~g} \mathrm{~g}^{-1}$, the reversible capacities of $365,292,242,215,181$ and $157 \mathrm{mAh} \mathrm{g}^{-1}$ were obtained at $0.1,0.2,0.5,1,2$ and $5 \mathrm{~A} \mathrm{~g} \mathrm{~g}^{-1}$, respectively. Even at a high current density of $10 \mathrm{~A} \mathrm{~g}^{-1}$, a relatively high capacity of $131 \mathrm{mAh}$ $\mathrm{g}^{-1}$ was still observed, $\sim 36 \%$ of that at $0.1 \mathrm{~A} \mathrm{~g}^{-1}$, indicating a high rate capability. When the current density was switched back to $0.2 \mathrm{~A} \mathrm{~g}^{-1}$, the capacity recovered to $289 \mathrm{mAh} \mathrm{g}^{-1}$, suggesting excellent stability. Though having a larger specific surface area and higher conductivity than HRGO300, RGO300 showed a much lower capacity of $251 \mathrm{mAh} \mathrm{g}^{-1}$ at $0.1 \mathrm{~A}$ $\mathrm{g}^{-1}$ and a lower rate capability $\left(\sim 17 \%\right.$ capacity retention at $10 \mathrm{~A} \mathrm{~g}^{-1}$ ) (Figure $3 \mathrm{~b}$ and Figure S7 in Supporting Information). The much better performance of HRGO300 than that of RGO300 is likely due to the synergism of enlarged interlayer distance and abundant pores on the partially reduced graphene oxide sheets, which can provide more capacitive sodium storage by accelerating the insertion/extraction of sodium ions and shortening the ion diffusion distance. The faster ion transfer process in HRGO300 can be evidenced by its smaller charge transfer resistance than that of RGO300. After cycling for ten cycles, the charge transfer resistance of both anodes clearly decreased as compared with the fresh state, which can be ascribed to the electrode activation process (Figure S8, Supporting Information). The energy storage capabilities of HRGO200 and HRGO400 were also tested, which were all lower than that of HRGO300 (Figure S9, Supporting Information). In this study, the $\mathrm{Na}^{+}$storage capability is 


\section{WILEY-VCH}

simultaneously affected by the oxygenous groups content, the interlayer distance and the conductivity of the products. The existence of oxygenous groups can increase the interlayer distance and contribute to capacity by redox reactions, which are desired for $\mathrm{Na}^{+}$storage. But too many oxygenous groups could reduce the number of available sites for $\mathrm{Na}^{+}$, hinder the $\mathrm{Na}^{+}$ transport and reduce the conductivity, which decrease the $\mathrm{Na}^{+}$storage capability. Hence, only the sample with optimized oxygenous groups amount can offer the best $\mathrm{Na}^{+}$storage performances. ${ }^{[14 \mathrm{~b}]}$ After 100 cycles at $0.1 \mathrm{~A} \mathrm{~g}^{-1}$, a high capacity of $276 \mathrm{mAh} \mathrm{g}^{-1}$ was maintained with a Coulombic efficiency of $\sim 100 \%$ (Figure $3 \mathrm{~d}$ ). At the high current density of $2 \mathrm{~A} \mathrm{~g}^{-1}$, the remaining capacity was $163 \mathrm{mAh} \mathrm{g}^{-1}$ after 3000 cycles with a Coulombic efficiency of $\sim 100 \%$, thereby indicating the high stability and reversibility of HRGO anodes (Figure 3e). As compared to reported carbon-based anode materials, the energy storage capability of HRGO300 is among the best for $\mathrm{Na}$ ion battery anodes. (Table S1, Supporting Information). 


\section{WILEY-VCH}

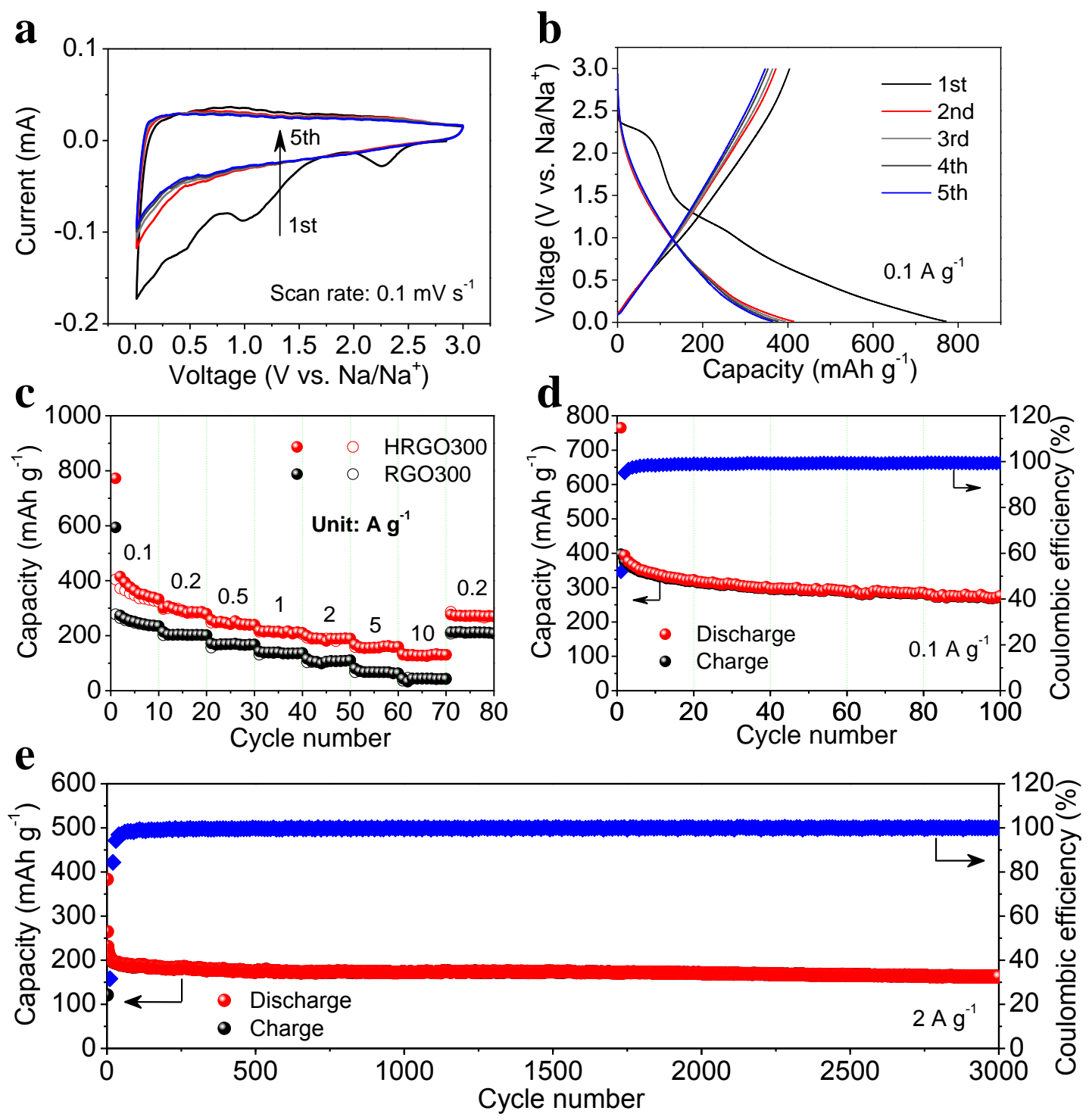

Figure 3. Electrochemical performance of HRGO300 and RGO300 electrodes; a) CV curves of HRGO300 at a scan rate of $0.1 \mathrm{mV} \mathrm{s}^{-1}, \mathrm{~b}$ ) galvanostatic charge-discharge profiles of HRGO300 at a current density of $0.1 \mathrm{~A} \mathrm{~g}^{-1}, \mathrm{c}$ ) rate performance of HRGO300 and RGO300 at different current densities, d) cycling performance of HRGO300 at a current density of $0.1 \mathrm{~A} \mathrm{~g}^{-}$ ${ }^{1}$, and e) cycling performance of HRGO300 at a current density of $2 \mathrm{~A} \mathrm{~g} \mathrm{~g}^{-1}$.

In order to understand the reaction kinetics of the electrodes, $\mathrm{CV}$ curves were measured at various scan rates (Figure S10, Supporting Information). The diffusion and capacitive contributions to current were analyzed according to $i=a v^{b}$, where the current (i) obeys a powerlaw relationship to the sweep rate $(v)$ and $a$ is a constant. For absolute diffusion-controlled process and capacitive-controlled process, the $b$ values are 0.5 and 1 , respectively. The $b$ value for the cathodic current at $1.5 \mathrm{~V}$ is 0.94 for HRGO300, which is superior to 0.86 for RGO300, suggesting that faster capacitive-controlled sodium storage occurs in HRGO300 anode. The 


\section{WILEY-VCH}

ratio of capacitive to diffusion contribution to the total capacity can be quantified by separating the capacitive- and diffusion-controlled currents under a certain potential. ${ }^{[9 a, 17,24]}$ Figure 4 exhibits the capacitive contribution to measured current for HRGO300 and RGO300 electrodes measured under different scan rates. For the HRGO300 electrode, the capacitive contribution can reach $59 \%$ at a slow scan rate of $0.1 \mathrm{mV} \mathrm{s}^{-1}$, which increases with increasing scan rate. The higher fraction of capacitive contribution under higher scan rate is ascribed to the rapid charging/discharging associated with capacitive processes. Under all the tested scan rates, the capacitive contribution in the HRGO300 electrode is higher than that of the RGO300 electrode, suggesting a faster sodium ion storage rate (Figure 4a). As known, the capacitive contribution includes the pseudocapacitive part and the surface adsorption part. Specially, in this study, the pseudocapacitive contribution consists of the surface redox reactions of oxygen-containing functional groups and pseudocapacitance owing to ion intercalation. The higher capacitive contribution of HRGO300 than RGO300 is attributed to the following three reasons: (i) the larger interlayer spacing of HRGO300 than RGO300 accelerates the ion insertion/extraction rate and thus increases the ion intercalation pseudocapacitance, (ii) the abundant holes on the sheets of HRGO300 can shorten the ion diffusion distance and provide more ion insertion/extraction sites, which further contributes to the intercalation pseudocapacitance, (iii) the abundant holes also provide more ion adsorption sites, which contributes to the fast surface adsorption capacitance and redox pseudocapacitance of surface oxygen-containing functional groups. ${ }^{[25]}$ A capacitive contribution as high as $77 \%$ at $0.6 \mathrm{mV} \mathrm{s}^{-1}$ was obtained for the HRGO300 electrode as illustrated in Figure 4b. 


\section{WILEY-VCH}

$\mathbf{a}$

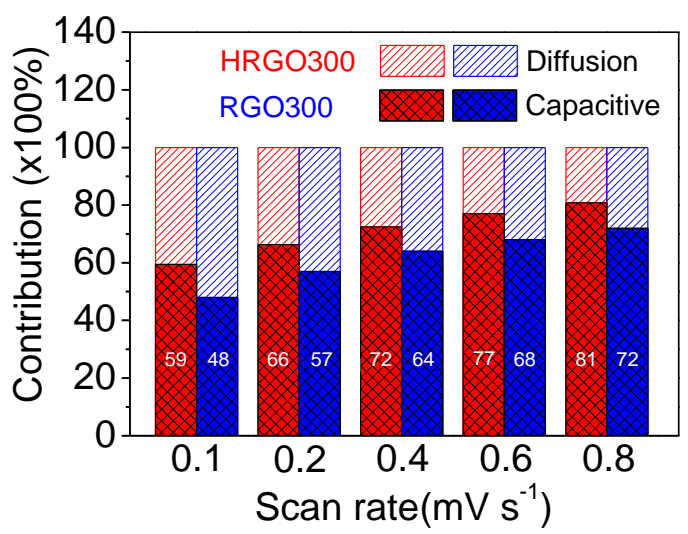

b

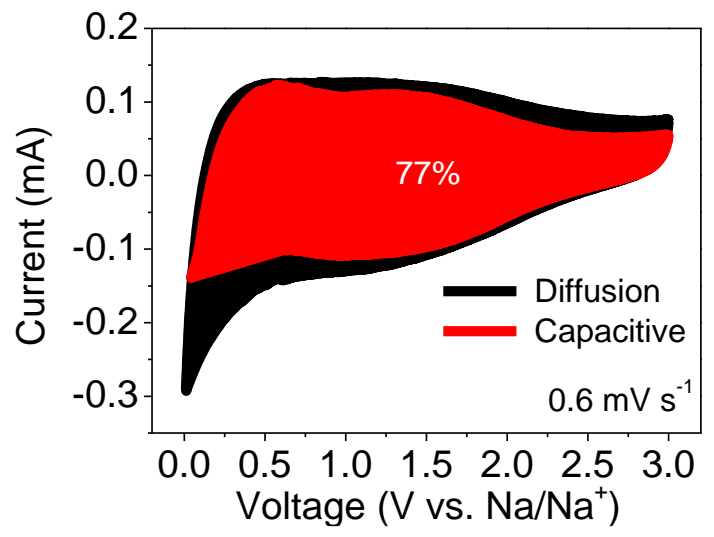

Figure 4. Electrochemical kinetics analysis of the electrodes. a) Capacity contributions from capacitive and diffusion-controlled processes for HRGO300 and RGO300 measured at different scan rates, b) CV curve of HRGO300 with the capacitive contribution at a scan rate of $0.6 \mathrm{mV}$ $\mathrm{s}^{-1}$.

In summary, a simple approach has been developed to increase the SIB anode performance by preparing partially reduced holey graphene oxide through low temperature-reduction of holey graphene oxide. The enlarged interlayer spacing $(0.434 \mathrm{~nm})$ ensures sufficient space for energy storage through sodium ions, while the pores on the partially reduced graphene oxide sheets enhance the insertion/extraction rate by shortening the ion transfer distance and providing more ion insertion/extraction sites. In addition, the residual oxygen-containing groups further contribute to the energy storage capability by redox reactions. As anode materials for SIBs, the HRGO prepared at $300{ }^{\circ} \mathrm{C}$ shows a high capacity of $365 \mathrm{mAh} \mathrm{g}^{-1}$ at $0.1 \mathrm{~A} \mathrm{~g}^{-1}$ with excellent rate capability and long cycle stability, much better than the control intact graphene sample prepared under the same temperature. This work provides a facile and scalable strategy to produce high performance anode materials for sodium ion-based energy storage devices.

\section{Experimental Section}

Preparation of oxygenous groups-interlayered HRGO: Graphene oxide (GO) was first prepared by a modified Hummers method. ${ }^{[26]}$ In a typical HRGO preparation procedure, $200 \mathrm{mg}$ GO was homogeneously dispersed in $100 \mathrm{~mL}$ of distilled water by sonication. Then, $10 \mathrm{~mL}$ of $30 \%$ $\mathrm{H}_{2} \mathrm{O}_{2}$ was added in the as-prepared $\mathrm{GO}$ aqueous dispersion and the mixture was heated to 


\section{WILEY-VCH}

$100{ }^{\circ} \mathrm{C}$ for $4 \mathrm{~h}$ under stirring. After cooling down to room temperature, the dispersion was purified by repetitive centrifuging and washing with distilled water. The resulting product was freeze-dried and then heated to $300{ }^{\circ} \mathrm{C}$ for $1 \mathrm{~h}$ in a quartz tube furnace in argon atmosphere at the heating rate of $10{ }^{\circ} \mathrm{C} \min ^{-1}$. Finally, the product, i.e. HRGO300, was produced after the furnace cooled down to room temperature. Similarly, HRGO200 and HRGO400 were also prepared. The control material RGO300 was prepared by directly heating the freeze-dried graphene oxide powder to $300{ }^{\circ} \mathrm{C}$ for $1 \mathrm{~h}$ under argon protection.

Structural characterization: Scanning electron microscopy (SEM, JSM-6701F at $10 \mathrm{kV}$ ), transmission electron microscopy (TEM, JEM-2100F operating at $200 \mathrm{kV}$ ), X-ray diffraction (XRD, Bruker D8 Advance A25, Co $K_{\alpha 1}$ radiation of $1.78897 \AA$ with a Fe filter of $0.02 \mathrm{~mm}$ thickness), Raman spectroscopy (Horiba JY LabRAM Evolution spectrometer with an excitation wavelength of $473 \mathrm{~nm}$ ), and X-ray photoelectron spectroscopy (XPS, PHI 5000 VersaProbe) were used for the characterization of the morphology, structure, and chemical composition of the products. The XRD data were converted to vs. Cu target. Nitrogen sorption isotherms were measured on Thermo Fisher Scientific Surfer Gas Adsorption Porosimeter at $77 \mathrm{~K}$. The SSA was calculated using the BET (Brunauer-Emmett-Teller) method based on the adsorption data in the linear relative pressure $\left(p / p_{0}\right)$ range of $0.05-0.3$. The pore size distribution was calculated from the corresponding adsorption branch of $\mathrm{N}_{2}$ isotherm by Horvath-Kawazoe method for micropore and Barrett-Joyner-Halenda method for meso- and macro-pore. The bulk conductivity of HRGO was measured under 6 MPa by a home-made device with Keithley 6430 as the source-measure unit. ${ }^{[10 c, 13 d]}$

Electrochemical measurements: The energy storage capability of as-prepared HRGO was assessed by employing HRGO as working electrode in 2025 coin-type cells. The working electrodes were made by homogeneously coating the slurry of $80 \mathrm{wt} \% \mathrm{HRGO}, 10 \mathrm{wt} \%$ super $\mathrm{P}$, and $10 \mathrm{wt} \%$ polytetrafluoroethylene (PTFE, $60 \mathrm{wt} \%$ dispersion in water) on copper foils. The loading mass of active material is about $1.23 \mathrm{mg} \mathrm{cm}^{-2}$. After drying at $100{ }^{\circ} \mathrm{C}$ in vacuum 


\section{WILEY-VCH}

for $12 \mathrm{~h}$, the cells were fabricated in a glove box with glass fibers as separators, sodium foils as counter electrodes, and 1.0 $\mathrm{M} \mathrm{NaClO}_{4}$ in ethylene carbonate (EC): dimethylcarbonate (DMC) (1:1 by volume) with $5 \%$ fluoroethylene carbonate additive as electrolyte. The galvanostatic charge-discharge and cyclic voltammetric tests were carried out in a voltage range of 0.01 to 3.0 V on a CT2001A cell test instrument (Wuhan LAND Electronic Co., Ltd) and a CHI 660E (Shanghai Chenhua instrument Co., Ltd) electrochemical workstation, respectively.

\section{Supporting Information}

Supporting Information is available from the Wiley Online Library or from the author.

\section{Acknowledgements}

J. Zhao and Y. Z. Zhang contributed equally to this work. Research reported in this publication has been supported by King Abdullah University of Science and Technology (KAUST) and by the Ministry of Education of Singapore.

Received: ((will be filled in by the editorial staff))

Revised: ((will be filled in by the editorial staff)) Published online: ((will be filled in by the editorial staff))

\section{References}

[1] a) S. Guo, J. Yi, Y. Sun, H. Zhou, Energy Environ. Sci. 2016, 9, 2978; b) D. Larcher, J. M. Tarascon, Nat. Chem. 2015, 7, 19; c) Y. Lu, Y. Lu, Z. Niu, J. Chen, Adv. Energy Mater. 2018, 8, 1702469; d) G.-L. Xu, R. Amine, A. Abouimrane, H. Che, M. Dahbi, Z.-F. Ma, I. Saadoune, J. Alami, W. L. Mattis, F. Pan, Z. Chen, K. Amine, Adv. Energy Mater. 2018, 8, 1702403.

[2] a) M. Lao, Y. Zhang, W. Luo, Q. Yan, W. Sun, S. X. Dou, Adv. Mater. 2017, 29, 1700622; b) W. Luo, F. Shen, C. Bommier, H. Zhu, X. Ji, L. Hu, Acc. Chem. Res. 2016, 49, 231; c) S. Wu, R. Ge, M. Lu, R. Xu, Z. Zhang, Nano Energy 2015, 15, 379.

[3] a) C. Fang, Y. Huang, W. Zhang, J. Han, Z. Deng, Y. Cao, H. Yang, Adv. Energy Mater. 2016, 6, 1501727; b) H. Kim, H. Kim, Z. Ding, M. H. Lee, K. Lim, G. Yoon, K. Kang, Adv. Energy Mater. 2016, 6, 1600943; c) H. Pan, Y.-S. Hu, L. Chen, Energy Environ. Sci. 2013, 6, 2338.

[4] a) H. Hou, X. Qiu, W. Wei, Y. Zhang, X. Ji, Adv. Energy Mater. 2017, 7, 1602898; b) Y. Xiao, S. H. Lee, Y.-K. Sun, Adv. Energy Mater. 2017, 7, 1601329.

[5] a) L. Peng, Y. Zhu, D. Chen, R. S. Ruoff, G. Yu, Adv. Energy Mater. 2016, 6, 1600025; b) J. Pang, A. Bachmatiuk, Y. Yin, B. Trzebicka, L. Zhao, L. Fu, R. G. Mendes, T. Gemming, Z. Liu, M. H. Rummeli, Adv. Energy Mater. 2018, 8, 1702093; c) K. Olszowska, J. Pang, P. S. Wrobel, L. Zhao, H. Q. Ta, Z. Liu, B. Trzebicka, A. Bachmatiuk, M. H. Rummeli, Synth. Met. 2017, 234, 53; d) W. Kai, Int. J. Electrochem. 


\section{WILEY-VCH}

Sci. 2017, 12, 8306; e) Q. Hao, J. Pang, Y. Zhang, J. Wang, L. Ma, O. G. Schmidt, Adv. Optical Mater. 2018, 6, 1700984; f) K. Wang, J. Pang, L. Li, S. Zhou, Y. Li, T. Zhang, Front. Chem. Sci. Eng. 2018, 12, 376.

[6] a) Y. Liu, F. Fan, J. Wang, Y. Liu, H. Chen, K. L. Jungjohann, Y. Xu, Y. Zhu, D. Bigio, T. Zhu, C. Wang, Nano Lett. 2014, 14, 3445; b) X. Zheng, J. Luo, W. Lv, D. W. Wang, Q. H. Yang, Adv. Mater. 2015, 27, 5388.

[7] a) H.-J. Choi, S.-M. Jung, J.-M. Seo, D. W. Chang, L. Dai, J.-B. Baek, Nano Energy 2012, 1, 534; b) M. F. El-Kady, Y. Shao, R. B. Kaner, Nat. Rev. Mater. 2016, 1, 16033; c) S. Han, D. Wu, S. Li, F. Zhang, X. Feng, Adv. Mater. 2014, 26, 849; d) F. Li, X. Jiang, J. Zhao, S. Zhang, Nano Energy 2015, 16, 488.

[8] a) X. Liu, D. Chao, D. Su, S. Liu, L. Chen, C. Chi, J. Lin, Z. X. Shen, J. Zhao, L. Mai, Y. Li, Nano Energy 2017, 37, 108; b) Y.-X. Wang, S.-L. Chou, H.-K. Liu, S.-X. Dou, Carbon 2013, 57, 202; c) Y. S. Yun, Y.-U. Park, S.-J. Chang, B. H. Kim, J. Choi, J. Wang, D. Zhang, P. V. Braun, H.-J. Jin, K. Kang, Carbon 2016, 99, 658; d) J. Zhang, C. Li, Z. Peng, Y. Liu, J. Zhang, Z. Liu, D. Li, Sci. Rep. 2017, 7, 4886.

[9] a) S. Li, J. Qiu, C. Lai, M. Ling, H. Zhao, S. Zhang, Nano Energy 2015, 12, 224; b) J. $\mathrm{Xu}, \mathrm{M}$. Wang, N. P. Wickramaratne, M. Jaroniec, S. Dou, L. Dai, Adv. Mater. 2015, 27, 2042; c) Y. Yan, Y.-X. Yin, Y.-G. Guo, L.-J. Wan, Adv. Energy Mater. 2014, 4, 1301584; d) F. Zhang, E. Alhajji, Y. Lei, N. Kurra, H. N. Alshareef, Adv. Energy Mater. 2018, 8, 1800353.

[10] a) S. Dutta, A. Bhaumik, K. C. W. Wu, Energy Environ. Sci. 2014, 7, 3574; b) J. Ni, Y. Li, Adv. Energy Mater. 2016, 6, 1600278; c) J. Zhao, Y. Jiang, H. Fan, M. Liu, O. Zhuo, X. Wang, Q. Wu, L. Yang, Y. Ma, Z. Hu, Adv. Mater. 2017, 29, 1604569.

[11] a) W. Chen, C. Chen, X. Xiong, P. Hu, Z. Hao, Y. Huang, Adv. Sci. 2017, 4, 1600500; b) H. Hou, L. Shao, Y. Zhang, G. Zou, J. Chen, X. Ji, Adv. Sci. 2017, 4, 1600243; c) W. Li, M. Zhou, H. Li, K. Wang, S. Cheng, K. Jiang, Energy Environ. Sci. 2015, 8, 2916; d) Y. Lin, X. Han, C. J. Campbell, J.-W. Kim, B. Zhao, W. Luo, J. Dai, L. Hu, J. W. Connell, Adv. Funct. Mater. 2015, 25, 2920; e) M. Wang, Y. Yang, Z. Yang, L. Gu, Q. Chen, Y. Yu, Adv. Sci. 2017, 4, 1600468; f) D. Xu, C. Chen, J. Xie, B. Zhang, L. Miao, J. Cai, Y. Huang, L. Zhang, Adv. Energy Mater. 2016, 6, 1501929; g) T. Yang, T. Qian, M. Wang, X. Shen, N. Xu, Z. Sun, C. Yan, Adv. Mater. 2016, 28, 539.

[12] L. M. H. Sun, J. Liang, Z. Zhao, C. Lee, H. Fei, M. Ding, J. Lau, M. Li, C. Wang, X. Xu, G. Hao, B. Papandrea, I. Shakir, B. Dunn, Y. Huang, X. Duan,, Science 2017, 356, 599.

[13] a) Z. J. Zhang, L. D. Xia, Sci. Adv. 2015, 1, e1500564; b) L. Hao, X. Li, L. Zhi, Adv. Mater. 2013, 25, 3899; c) J. P. Paraknowitsch, A. Thomas, Energy Environ. Sci. 2013, 6, 2839; d) J. Zhao, H. Lai, Z. Lyu, Y. Jiang, K. Xie, X. Wang, Q. Wu, L. Yang, Z. Jin, Y. Ma, J. Liu, Z. Hu, Adv. Mater. 2015, 27, 3541; e) J. Zhang, W. Lv, Y. Tao, Y.-B. He, D.-W. Wang, C.-H. You, B. Li, F. Kang, Q.-H. Yang, Energy Storage Mater. 2015, 1, 112.

[14] a) Y. Cao, L. Xiao, M. L. Sushko, W. Wang, B. Schwenzer, J. Xiao, Z. Nie, L. V. Saraf, Z. Yang, J. Liu, Nano Lett. 2012, 12, 3783; b) Y. Wen, K. He, Y. Zhu, F. Han, Y. Xu, I. Matsuda, Y. Ishii, J. Cumings, C. Wang, Nat. Commun. 2014, 5, 4033.

[15] K. Tang, L. Fu, R. J. White, L. Yu, M.-M. Titirici, M. Antonietti, J. Maier, Adv. Energy Mater. 2012, 2, 873.

[16] a) H. Chen, M. B. Müller, K. J. Gilmore, G. G. Wallace, D. Li, Adv. Mater. 2008, 20, 3557; b) J. Yan, Y. Xiao, G. Ning, T. Wei, Z. Fan, RSC Adv. 2013, 3, 2566; c) X. Han, M. R. Funk, F. Shen, Y. C. Chen, Y. Li, C. J. Campbell, J. Dai, X. Yang, J. W. Kim, Y. Liao, J. W. Connell, V. Barone, Z. Chen, Y. Lin, L. Hu, ACS Nano 2014, 8, 8255; d) Y. Yoon, K. Lee, C. Baik, H. Yoo, M. Min, Y. Park, S. M. Lee, H. Lee, Adv. Mater. 2013, 25, 4437. 


\section{WILEY-VCH}

[17] J. Zhang, D.-W. Wang, W. Lv, S. Zhang, Q. Liang, D. Zheng, F. Kang, Q.-H. Yang, Energy Environ. Sci. 2017, 10, 370.

[18] a) K. A. Cychosz, R. Guillet-Nicolas, J. Garcia-Martinez, M. Thommes, Chem. Soc. Rev. 2017, 46, 389; b) D. H. E. K.S. Sing, R.A.W. Haul, L. Moscou, R.A. Pierotti, J. Rouquerol, T. Siemieniewska,, Pure Appl. Chem. 1985, 57, 603.

[19] L. J. Wang, M. F. El-Kady, S. Dubin, J. Y. Hwang, Y. Shao, K. Marsh, B. McVerry, M. D. Kowal, M. F. Mousavi, R. B. Kaner, Adv. Energy Mater. 2015, 5, 1500786.

[20] a) J. Yang, X. Zhou, D. Wu, X. Zhao, Z. Zhou, Adv. Mater. 2017, 29, 1604108; b) Y. Li, Y.-S. Hu, M.-M. Titirici, L. Chen, X. Huang, Adv. Energy Mater. 2016, 6, 1600659.

[21] H. Hou, C. E. Banks, M. Jing, Y. Zhang, X. Ji, Adv. Mater. 2015, $27,7861$.

[22] S. Wang, L. Xia, L. Yu, L. Zhang, H. Wang, X. W. D. Lou, Adv. Energy Mater. 2016, 6,1502217

[23] a) R. Hao, Y. Yang, H. Wang, B. Jia, G. Ma, D. Yu, L. Guo, S. Yang, Nano Energy 2018, 45, 220; b) S. Liu, J. Zhou, H. Song, Adv. Energy Mater. 2018, 8, 1800569.

[24] Q. Liang, Y. Zheng, C. Du, Y. Luo, J. Zhang, B. Li, Y. Zong, Q. Yan, Small Methods 2017, 1, 1700304 .

[25] a) V. Augustyn, P. Simon, B. Dunn, Energy Environ. Sci. 2014, 7, 1597; b) C. Chen, Y. Wen, X. Hu, X. Ji, M. Yan, L. Mai, P. Hu, B. Shan, Y. Huang, Nat. Commun. 2015, 6, 6929; c) Z. Chen, V. Augustyn, X. Jia, Q. Xiao, B. Dunn, Y. Lu, ACS Nano 2012, 6, 4319; d) Z. Hu, L. Wang, K. Zhang, J. Wang, F. Cheng, Z. Tao, J. Chen, Angew. Chem. Int. Ed. 2014, 53, 12794; e) R. Sun, Q. Wei, J. Sheng, C. Shi, Q. An, S. Liu, L. Mai, Nano Energy 2017, 35, 396.

[26] W. S. Hummers, R. E. Offeman, J. Am. Chem. Soc. 1958, 80, 1339. 


\section{WILEY-VCH}

Partially reduced holey graphene oxide is prepared by low temperature-reduction of holey graphene oxide. The large interlayer distance, short ion transfer distance, increased ion insertion/extraction sites and residual oxygenous groups endow partially reduced holey graphene oxide with high sodium ion storage capability. This approach provides a facile and scalable strategy to produce high performance anode materials for sodium ion batteries.

\section{Keyword}

Holey graphene oxide; Anode material; Energy storage; Sodium-ion batteries; Oxygenous groups

Jin Zhao, Yi-Zhou Zhang, Fan Zhang, Hanfeng Liang, Fangwang Ming, Husam N. Alshareef* and Zhiqiang Gao*

Partially Reduced Holey Graphene Oxide as High-Performance Anode for Sodium-Ion

\section{Batteries}

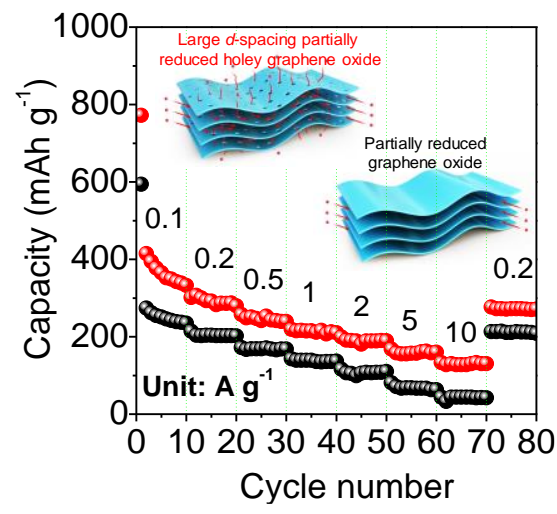

